\title{
A Dissolution Test for Finasteride in Immediate-Release Capsules
}

\author{
Olimpia Maria Martins Santos*, Ana Laura Araújo Santos, \\ Gislaine Ribeiro Pereira, Rudy Bonfilio, and \\ Magali Benjamim de Araújo \\ Faculdade de Ciências Farmacêuticas, Universidade Federal de Alfenas, Rua Gabriel \\ Monteiro da Silva, 700, 37130-000, Alfenas-MG, Brazil
}

e-mail:olimpia_martins@yahoo.com.br

\begin{abstract}
In vitro dissolution tests for solid oral dosage forms are extremely important to ensure the quality of these products. However, no dissolution test has been reported for finasteride (FNS) in immediate-release capsules. The aims of this work were to optimize a dissolution method for FNS capsules, validate the analytical method, and evaluate three different commercial products. The best in vitro dissolution profile was achieved using water as the dissolution medium with a basket stirrer at $100 \mathrm{rpm}$. The quantitative determination was performed by high performance liquid chromatography (HPLC) at $210 \mathrm{~nm}$. All validation parameters were satisfactory. The application of the method to commercial products showed the discriminatory power of the dissolution method. Because there is no monograph for FNS in capsules, this study illustrates the importance of an official dissolution test for FNS in capsules and the need to standardize the composition of the excipients contained in these commercial products.
\end{abstract}

\section{INTRODUCTION}

$\mathrm{n}$ vitro dissolution tests for immediate-release solid oral dosage forms, such as tablets and capsules, are ex-

tremely important because these tests are essential to evaluate the lot-to-lot quality of a drug product, to guide the development of new formulations, and to ensure continuing product quality and performance after certain changes (e.g., formulation, manufacturing process, site of manufacture, and scale-up of the manufacturing process) (1). For these reasons, progressively more emphasis has been placed on dissolution testing within the pharmaceutical industry and by regulatory authorities (2).

Despite the importance of dissolution tests in ensuring the quality of medicines, several drug products that are widely commercialized throughout the world lack official dissolution tests. Finasteride (FNS) is a relevant example because no dissolution test is reported in the literature or in pharmacopeias for immediate-release capsules of this medication. FNS capsules are widely marketed and are prepared for medical reasons (e.g., avoiding an excipient to which a patient is allergic or obtaining a dosage level that is not marketed).

FNS is $N$-(1,1-dimethylethyl)-3-oxo-4-aza- $5 \alpha$-androst1-ene-17 $\beta$-carboxamide (Figure 1), a specific competitive inhibitor of steroid type-II $5 \alpha$-reductase, an intracellular enzyme that converts testosterone to dihydrotestosterone (DHT) and is widely used for the treatment of benign prostatic hyperplasia (BPH) (3), prostate cancer (4), and androgenetic alopecia (5-8). A daily dose of $5 \mathrm{mg}$ has been used for the treatment of BPH and prostate cancer, and a 1-mg dose has been used for the treatment of androgenetic alopecia. FNS has a molecular formula of
$\mathrm{C}_{23} \mathrm{H}_{36} \mathrm{~N}_{2} \mathrm{O}_{2}$, a molecular weight of 372.6 , and a $\log P_{\mathrm{o} / \mathrm{w}}$ of 3.03. According to the Biopharmaceutics Classification System (BCS), FNS is a Class 2 drug having low solubility and high permeability (9).

Multiple analytical procedures have been reported for the analysis of FNS in pharmaceutical preparations when it is present as a single active ingredient or in combination dosage forms, using spectrophotometry (10-12), thin-layer chromatography $(13,14)$, infrared spectrophotometry (15), polarography (16), voltammetry (17), gas chromatography (18), gas chromatography-mass spectrometry (19), high performance liquid chromatography (HPLC) (20-23), and ultra-high performance liquid chromatography $(\mathrm{UH}-$ $\operatorname{PLC}(24,25)$.

Because of the importance of in vitro dissolution tests for immediate-release solid oral dosage forms and the lack of a dissolution test for FNS compounded capsules, the aims of this work were to optimize a dissolution method

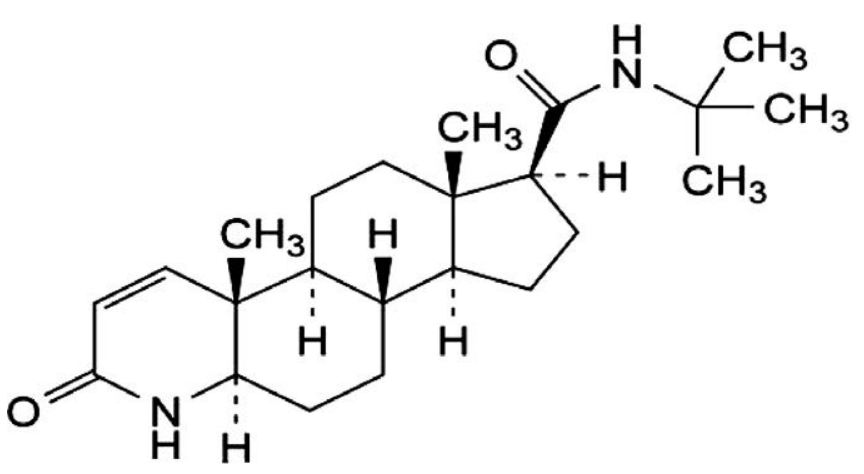

Figure 1. Chemical structure of finasteride. 
for FNS capsules, validate the analytical method used for the quantification of the samples, and demonstrate the applicability of the dissolution test by evaluating three different commercial products.

\section{EXPERIMENTAL}

\section{Chemicals and Reagents}

All reagents were of analytical grade. Potassium dihydrogenphosphate $\left(\mathrm{KH}_{2} \mathrm{PO}_{4}\right)$, phosphoric acid, sodium hydroxide, sodium acetate, and hydrochloric acid were purchased from Vetec (Rio de Janeiro, Rio de Janeiro, Brazil). FNS reference substance (assigned purity 99.7\%) was acquired from USP (Rockville, Maryland, USA). Acetonitrile was of HPLC grade and was acquired from both Vetec (Rio de Janeiro, Rio de Janeiro, Brazil) and Burdick \& Jackson (Muskegon, Michigan, USA). Reverse osmosis water was obtained using a water purifier OS 10 LTH (Gehaka, São Paulo, São Paulo, Brazil) and was used to prepare dissolution media. HPLC grade water used in the mobile phase was prepared with a Milli-Q system (Millipore, Bedford, Massachusetts, USA).

FNS capsules that contained $5 \mathrm{mg}$ of the active component were used in this study and were supplied from three compounding pharmacies, codified as A, B, and C. In addition, a placebo mixture containing the same quantitative composition as the capsules was obtained from the compounding pharmacies. The placebo mixture contained the following inactive excipients: aerosil, sodium lauryl sulfate, talc, starch, and lactose hydrous powder (Product A); talc, starch, and lactose hydrous powder (Product B); and magnesium stearate, aerosil, sodium lauryl sulfate, talc, starch, and lactose hydrous powder (Product C).

The FNS reference drug product (Proscar tablets, lot 300060) was purchased from Merck Sharp \& Dohme (Whitehouse Station, New Jersey, USA). This product contained $5 \mathrm{mg}$ of the active component and the following pharmaceutical grade excipients: cellulose, microcrystalline, docusate sodium, lactose monohydrate, magnesium stearate, pregelatinized maize starch, sodium starch glycolate type $A$, yellow iron oxide, hyprolose, indigo carmine aluminium lake, hypromellose, talc, and titanium dioxide.

\section{Instrumentation}

Dissolution tests were performed in an Electrolab TDT08L multi bath $(n=8)$ dissolution test system (Mumbai, Maharashtra, India) equipped with Apparatus 1 (basket) and 2 (paddle) in accordance with USP General Chapter $<711>(26)$.

The HPLC was a Shimadzu series LC-10A (Kyoto, Kyoto, Japan) consisting of LC-10ADVP pump, CLASS-VP 5.02 integration system software, DGU-14A degasser, 7725i manual injector with a 20- $\mu \mathrm{L}$ loop (Rheodyne, Rohnert Park, California, USA), SPD-10AVP integrated UV detector, LC-10AVP low-pressure gradient valve, CTO-10AVP column oven, and SCL-10AVP controller.
The following equipment was also used: digital $\mathrm{pH}$ meter model PA 200 (Marconi S.A., Piracicaba, São Paulo, Brazil), ultrasonic bath model USC 2800A (Unique, Indaiatuba, São Paulo, Brazil), analytical balance Acculab model ALC 210.4 (São Paulo, São Paulo, Brasil), and vacuum filtration system (Millipore, Bedford, Massachusetts, USA).

\section{Solutions}

All dissolution media used in this study (water, $0.1 \mathrm{M}$ hydrochloric acid, $\mathrm{pH} 4.5$ acetate buffer, and $\mathrm{pH} 6.8$ phosphate buffer) were degassed at $41^{\circ} \mathrm{C}$ in an ultrasonic bath for $20 \mathrm{~min}$ prior to use. The $0.1 \mathrm{M}$ hydrochloric acid solution was prepared by adding $8.5 \mathrm{~mL}$ of hydrochloric acid to $1.0 \mathrm{~L}$ of water. The $\mathrm{pH} 4.5$ sodium acetate buffer $(0.05 \mathrm{M})$ was prepared by adding $5.98 \mathrm{~g}$ of sodium acetate trihydrate and $3.32 \mathrm{~mL}$ of glacial acetic acid to $2.0 \mathrm{~L}$ of water. The $\mathrm{pH} 6.8$ potassium phosphate buffer $(0.072 \mathrm{M})$ was prepared by adding $13.609 \mathrm{~g}$ of potassium dihydrogenphosphate and $22.38 \mathrm{~mL}$ of $2.0 \mathrm{M}$ sodium hydroxide to $2.0 \mathrm{~L}$ of water.

The stock standard solution of FNS at $100 \mu \mathrm{g} / \mathrm{mL}$ was prepared in a $100-\mathrm{mL}$ volumetric flask by dissolving an accurately weighed amount $(10.0 \mathrm{mg})$ of FNS standard (99.7\% purity) in the mobile phase. Working standard solutions were prepared immediately before use by diluting the corresponding stock solutions to appropriate concentration levels using potassium phosphate buffer, sodium acetate buffer, hydrochloric acid, or purified water.

Sample solutions were prepared by placing one capsule in each vessel $(n=6)$ containing the dissolution medium $(500 \mathrm{~mL})$ at a temperature of $37 \pm 0.5^{\circ} \mathrm{C}$. Samples were collected at the end of the specified time and filtered through quantitative VETEC filter paper. For the HPLC analysis, standard and samples were filtered through a 0.45- $\mu \mathrm{m}$ PTFE membrane filter (Millipore, Bedford, Massachusetts, USA) prior to injection into the HPLC system.

\section{Chromatographic Conditions}

The chromatographic separation was achieved at $30^{\circ} \mathrm{C}$ on a Lichrospher reversed-phase C8 $(150 \times 4.6 \mathrm{~mm}, 5 \mu \mathrm{m})$ endcapped column (MetaChem Technologies Inc., Torrance, California, USA) using acetonitrile/water (70:30, v/v) as the mobile phase at a flow rate of $1.0 \mathrm{~mL} / \mathrm{min}$. The mobile phase was prepared daily, filtered through a $0.45-\mu \mathrm{m}$ membrane filter (Millipore, Bedford, Massachusetts, USA), and degassed using the degasser of the chromatographic system prior to use. The detector wavelength was set at $210 \mathrm{~nm}$, and the injection volume was $20 \mu \mathrm{L}$.

\section{Finasteride Solubility}

The solubility of finasteride was expressed in terms of $\log D$. The partition coefficient of an uncharged compound in the aqueous and octanol phases is called log $P$ (for partition). When a given $\mathrm{pH}$ is used, the resulting 
partition coefficient is called $\log D$ (for distribution). In this work, theoretical values for finasteride $\log D$ between $\mathrm{pH}$ 1 and 13 were calculated using ACD Lab software (ACD/ Structure Designer, version 12.0, Advanced Chemistry Development, Toronto ON, Canada).

\section{Dissolution Test Optimization}

Because there is no reference product in capsule form, the dissolution experimental conditions were established with only one of the three products used in this work (capsule C). Initially, water, $0.1 \mathrm{M} \mathrm{HCl}, \mathrm{pH} 4.5$ acetate buffer, and $\mathrm{pH} 6.8$ potassium phosphate buffer were tested as dissolution media using a basket stirrer at a speed of 50 rpm. With water as the dissolution medium, the influence of rotation speed of the basket stirrer on the FNS capsule release was checked at 50,75, and $100 \mathrm{rpm}$. Finally, the performance of the basket stirrer versus the paddle stirrer was compared at $100 \mathrm{rpm}$ on the FNS release in water. When the paddle stirrer was tested, a wire helix (sinker) was used around the capsules to avoid floating of the capsules, as recommended by the USP (26). A volume of 500 $\mathrm{mL}$ was used in the dissolution vessels, and the stabilization temperature was $37.0 \pm 0.5^{\circ} \mathrm{C}$. Samples $(2 \mathrm{~mL})$ were withdrawn from the dissolution medium at 3, 9, 15, 20, 30, 45 , and $60 \mathrm{~min}$, followed by immediate replacement. The samples were analyzed by HPLC, and the analytical results were employed for the acquisition of dissolution profiles. Six samples were assayed.

\section{Method Validation}

After optimization of the dissolution test, the analytical method was validated according to the USP and the International Conference on Harmonization (ICH) requirements $(26,27)$. To demonstrate whether the method was adequate for dissolution test purposes, the method was validated by the determination of stability, selectivity, linearity, precision, accuracy, detection limit, quantitation limit, and robustness parameters.

\section{Stability}

Working standard solutions at $10.0 \mu \mathrm{g} / \mathrm{mL}$ of FNS in water were prepared in triplicate, filtered, and immediately injected into the HPLC system (time zero). The same solutions were maintained at room temperature for $24 \mathrm{~h}$ and evaluated again. Then the results obtained after 24 $\mathrm{h}$ were compared with those obtained at time zero. The acceptance criterion is an analytical response after $24 \mathrm{~h}$ between $98 \%$ and $102 \%$ of the value obtained at time zero.

To evaluate the FNS stability under dissolution test conditions, three samples of each product (A, B, and C) containing $5 \mathrm{mg}$ of FNS were transferred to separate vessels containing $500 \mathrm{~mL}$ of water at $37 \pm 0.5^{\circ} \mathrm{C}$ and stirred for $45 \mathrm{~min}$ at $100 \mathrm{rpm}$ using basket stirrers. Aliquots were collected, filtered, and immediately injected into the HPLC (time zero). The same solutions were maintained at room temperature for $2 \mathrm{~h}$ and evaluated again. For each solution, the results obtained after $2 \mathrm{~h}$ were compared with the results obtained at time zero. An analytical response after $2 \mathrm{~h}$ between $98 \%$ and $102 \%$ of the response obtained at time zero is acceptable.

\section{Selectivity}

For determination of selectivity, the placebo sample and capsule shell were transferred to separate vessels containing $500 \mathrm{~mL}$ of water at $37 \pm 0.5^{\circ} \mathrm{C}$ and stirred for $45 \mathrm{~min}$ at $100 \mathrm{rpm}$ using the basket stirrer as the apparatus. Aliquots were collected, and the interference of the placebo mixture of each formulation was evaluated by HPLC.

\section{Linearity}

Linearity was evaluated by regression analysis of the FNS working standard solutions at six concentrations in triplicate ranging from 0.5 to $15.0 \mu \mathrm{g} / \mathrm{mL}(0.5,1.0,5.0,8.0$, 10.0 , and $15.0 \mu \mathrm{g} / \mathrm{mL}$ ) prepared in water on three consecutive days $(n=3)$. The calibration equations and the correlation coefficient were calculated. A correlation coefficient of $\geq 0.999$ and a relative standard deviation (RSD) of each point $(n=9)$ less than $2 \%$ were the acceptance criteria (26).

\section{Accuracy and Precision}

The accuracy was evaluated through a recovery test using known amounts of FNS reference substance added to the placebo. A stock solution containing $5 \mathrm{mg} / \mathrm{mL}$ of FNS was prepared in the mobile phase. Aliquots of 0.5, 1.0 , and $1.5 \mathrm{~mL}$ of this solution were added to vessels containing the dissolution medium for a total volume of $500 \mathrm{~mL}$ (final concentrations were 5,10 , and $15 \mu \mathrm{g} / \mathrm{mL}$, which correspond to $50 \%, 100 \%$, and $150 \%$ of the target concentration). Placebo samples containing the same excipient composition as the $5 \mathrm{mg}$ capsule products (A, B, and $C$ ) were then transferred to each vessel. The samples were maintained at $37 \pm 0.5^{\circ} \mathrm{C}$ and stirred at $150 \mathrm{rpm}$ for 1 h. Subsequently, aliquots from each vessel were collected and analyzed. These studies were performed in triplicate on three different days. The percent recoveries were calculated according to:

$$
R(\%)=\frac{\left(C_{\text {measured }}\right)}{\left(C_{\text {added }}\right)} \times 100 \%
$$

where $C_{\text {measured }}$ is the sample concentration obtained from the HPLC method at each concentration level and $C_{\text {added }}$ is the theoretical concentration at each concentration level. The acceptance criterion was a recovery between $95.0 \%$ and $105.0 \%$ (26).

The same solutions used in the accuracy test were analyzed to ensure the precision of the method. Intra- and interday precision were established based on RSD of the results (27). The acceptance criterion was an RSD less than $5.0 \%(26)$. 
Table 1. Factors and Levels for Robustness Studies and Experimental Conditions ${ }^{a}$

\begin{tabular}{|c|c|c|c|c|c|c|c|c|c|c|}
\hline \multirow[b]{2}{*}{ Selected factor } & \multirow[b]{2}{*}{ Nominal Conditions } & \multirow[b]{2}{*}{ Alternative Conditions } & \multicolumn{8}{|c|}{ Experimental Conditions } \\
\hline & & & 1 & 2 & 3 & 4 & 5 & 6 & 7 & 8 \\
\hline Stirring speed & $100 \mathrm{rpm}(\mathrm{A})$ & 95 rpm (a) & A & A & A & A & $\mathrm{a}$ & $\mathrm{a}$ & a & a \\
\hline Dissolution medium degassing & Yes (B) & No (b) & B & B & $\mathrm{b}$ & $b$ & B & B & $\mathrm{b}$ & $b$ \\
\hline Sample filtration & Yes (C) & No (c) & C & C & C & $C$ & C & C & C & C \\
\hline Time of dissolution test & $45 \min (D)$ & $47 \min (d)$ & D & $\mathrm{D}$ & d & $d$ & d & $d$ & D & D \\
\hline Dissolution medium temperature & $37^{\circ} \mathrm{C}(\mathrm{E})$ & $35^{\circ} \mathrm{C}(\mathrm{e})$ & $E$ & $\mathrm{e}$ & $E$ & e & e & $E$ & e & $\mathrm{E}$ \\
\hline Exposure to light & Yes (F) & No (f) & $\mathrm{F}$ & $f$ & $f$ & $\mathrm{~F}$ & $\mathrm{~F}$ & $f$ & $f$ & $\mathrm{~F}$ \\
\hline Sampling point & upper (G) & down (g) & G & g & $g$ & G & $g$ & G & G & g \\
\hline Observed results & & & $\mathrm{s}$ & $\mathrm{t}$ & $\mathrm{u}$ & v & w & $x$ & $y$ & z \\
\hline
\end{tabular}

${ }^{a}$ According to the Youden and Steiner robustness test (2). Upper case letters ( $\left.A-G\right)$ represent nominal conditions; lower case letters ( $a-g$ ) represent alternative conditions.

\section{Detection and Quantitation Limits}

The detection limit (DL) and quantitation limit (QL) were obtained from

$$
\begin{gathered}
\mathrm{DL}=3(S D / a) \\
\mathrm{QL}=10(S D / a)
\end{gathered}
$$

where $S D$ is the intersection standard deviation and $a$ is the slope of the calibration curves obtained in the linearity study (27).

\section{Robustness}

The robustness was evaluated by analyzing the data after varying seven factors according to the Youden and Steiner robustness test (29). The percentage of drug release was calculated for each experiment by comparing the sample peak areas with those obtained from the FNS standard solution at the same concentration level. The following factors were probed: stirring speed, degassing of the dissolution medium, sample filtration, time of dissolution test, temperature of the dissolution medium, exposure to light, and sampling point. The nominal values of the factors are denoted by $A, B, C, D, E, F$, and $G$, and their alternative values are denoted by the corresponding lowercase letters $\mathrm{a}, \mathrm{b}, \mathrm{c}, \mathrm{d}, \mathrm{e}, \mathrm{f}$, and g. Eight experiments were conducted, as indicated in Table 1. From these results, the effect of each factor was estimated by obtaining the difference of the averages of the four analyses that have the nominal value (uppercase letter) and the four analyses with the alternative value (lowercase letter). Considering the standard deviation of the eight results, effect values higher than the $\mathrm{SD} \sqrt{2}$ criterion (standard deviation multiplied by the square root of two) were considered significant, and the method is sensitive to the changes in the specific factor.

\section{Assay of Pharmaceutical Products}

After determination of the best dissolution conditions as well as analytical method validation, three different commercial products ( $A, B$, and $C$ ) were evaluated. The dissolution experimental conditions were water as the dissolution medium and a basket stirrer as the apparatus, stirring at a rate of $100 \mathrm{rpm}$. Two milliliters of dissolution medium (controlled at $37.0 \pm 0.5^{\circ} \mathrm{C}$ ) were sampled after $3,9,15,20,30,45$, and $60 \mathrm{~min}$, followed by immediate replacement and then analyzed by HPLC. In these tests, the last sampling point was 60 min because the USP (26) recommends a procedure duration of 30-60 min for immediate-release dosage forms.

The same methodology was applied to reference product Proscar in 5-mg tablets. Comparisons between the dissolution profiles of the commercial products and the dissolution profile obtained from the reference product (reference $\times A$, reference $\times B$ and reference $\times C$ ) were carried out. The similarity of the dissolution profiles was determined by the similarity factor $\left(f_{2}\right)$, calculated as follows:

$$
f_{2}=50 \cdot \log \left\{\left[1+\frac{1}{n} \sum_{t=1}^{n}\left(R_{t}-T_{t}\right)^{2}\right]^{-0.5} \times 100\right\}
$$

where $R_{t}$ is the percentage of reference drug dissolved at each time point, $T_{t}$ is the percentage of test product dissolved in each time point, and $n$ is the number of sampling time points.

For curves to be considered similar, $f_{2}$ values should be close to 100 . Generally, $f_{2}$ values greater than 50 (i.e., 50-100) ensure equivalence of the two curves (1).

\section{RESULTS AND DISCUSSION \\ Optimization of HPLC Conditions}

The chromatographic conditions were previously optimized to yield an adequate analytical performance in a short run time. After several preliminary experiments, the following parameters were considered adequate for our purpose: acetonitrile/water (70:30, v/v) mobile phase, a flow rate of $1.0 \mathrm{~mL} / \mathrm{min}$, a column temperature of $30^{\circ} \mathrm{C}$, 


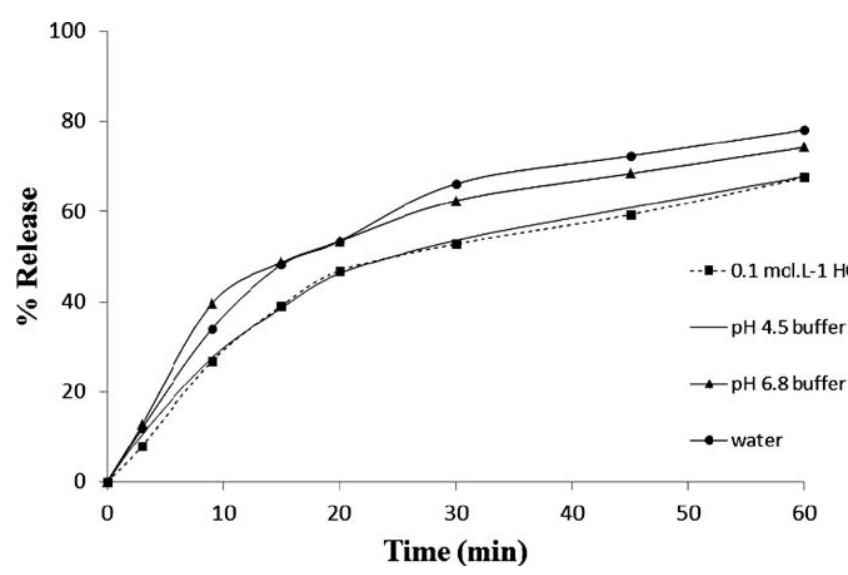

Figure 2. Dissolution profiles of 5-mg FNS capsules using $0.1 \mathrm{M} \mathrm{HCl}$, acetate buffer ( $p H 4.5 ; 0.05 \mathrm{M})$, phosphate buffer ( $\mathrm{pH} 6.8 ; 0.072 \mathrm{M})$, and water as dissolution media. A paddle stirrer was used at $50 \mathrm{rpm}$. Samples were analyzed by HPLC.

a reversed-phase $C 8(150 \times 4.6 \mathrm{~mm}, 5 \mu \mathrm{m})$ endcapped column, a UV detection wavelength at $210 \mathrm{~nm}$, and an injection volume of $20 \mu \mathrm{L}$.

To confirm whether the chromatographic parameters were consistent with the literature, a system suitability test was carried out by injecting six replicates of a working standard solution containing $10.0 \mu \mathrm{g} / \mathrm{mL}$ of FNS in water. The following results were found: retention time of 3.09 min, theoretical plates greater than 5800, a retention factor of 3.29, and FNS peak asymmetry of 1.12. The relative standard deviation of the peak area was $0.68 \%$. All parameters were therefore consistent with USP recommendations (26).

\section{Dissolution Test Optimization}

Because there is no reference product in capsule form, the dissolution experimental conditions were established by submitting only one of the three capsule products that were used in this work. We selected product $C$ because it had an excipient composition more similar to the reference product.

In general, mild conditions should be maintained during dissolution testing to allow maximum discriminating power (30). In this study, the dissolution test was optimized in terms of dissolution medium, rotation speed, and basket (type 1)/paddle (type 2) stirring apparatus to obtain a slow dissolution of the products at the first sampling points, which certainly will result in a suitably discriminative test. In addition, the dissolution test was one that can produce a satisfactory release of the products at the last sampling points according to FDA specifications (1) that state that only one measurement should be considered after $85 \%$ dissolution for comparison of dissolution profiles using the simple model-independent approach.

Regarding the dissolution medium, $500 \mathrm{~mL}$ was used because this volume was sufficient to dissolve the 5-mg

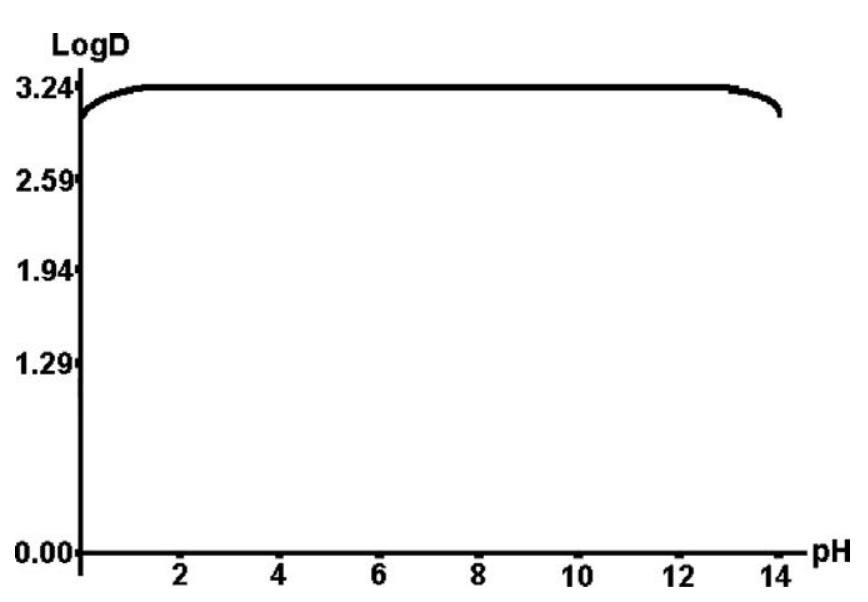

Figure 3. Log D for FNS calculated by ACD Lab software.

dosage units. In the optimization of the dissolution medium composition (using the basket stirrer apparatus with a stirring speed of $50 \mathrm{rpm}$ ), $0.1 \mathrm{M} \mathrm{HCl}$ and $\mathrm{pH} 4.5$ acetate buffer were not considered "ideal" because the FNS capsules had a low and constant dissolution profile in these media without reaching a maximum in up to $60 \mathrm{~min}$ (Figure 2). However, pH 6.8 phosphate buffer and the basket stirrer apparatus at a stirring speed of $50 \mathrm{rpm}$ allowed a slow FNS release at the first sampling points and a high drug release at $60 \mathrm{~min}$. Phosphate buffer (pH 6.8) as the dissolution medium would probably result in a suitably discriminative test (Figure 2).

The use of water as a dissolution medium is discouraged because test conditions such as $\mathrm{pH}$ and surface tension can vary depending on the water source and day and may change during the dissolution test itself. However, the aqueous solubility of FNS was almost constant between pH 1 and 13 (Figure 3). The phosphate buffer was therefore replaced by water because water is less expensive, readily available, easily disposed of, environmentally

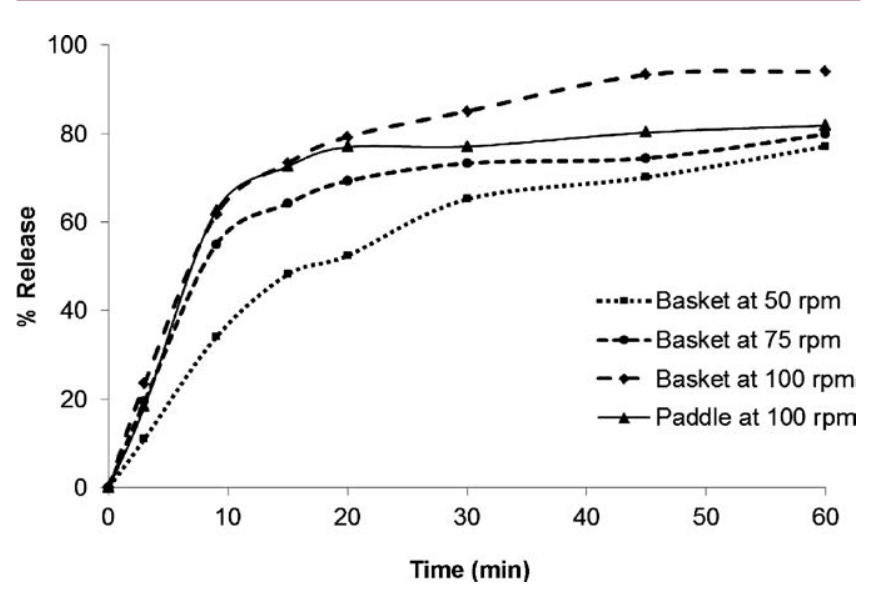

Figure 4. Optimization of rotation speed and stirring apparatus. Water was used as the dissolution medium and the samples were analyzed by HPLC. 
Table 2. Stability Studies

\begin{tabular}{lcc}
\hline Initial (100.0\%) & \% Recovery (after 2 h) & \% Recovery (after 24 h) \\
\hline Standard & - & 99.2 \\
\hline Sample A & 99.5 & - \\
Sample B & 99.8 & - \\
Sample C & 99.4 & - \\
\hline
\end{tabular}

friendly, and suitable for products with a release rate independent of $\mathrm{pH}$ value of the medium. Therefore, water was chosen as the best dissolution medium because results similar to those observed with phosphate buffer were obtained.

The influence of the rotation speed of the basket stirrer (at 50, 75, and $100 \mathrm{rpm}$ ) on the FNS capsules release was evaluated with water as the dissolution medium. The experimental results show the best dissolution rate at the last sampling points at a 100-rpm rotation speed (Figure 4). This result is expected because an increase of the apparatus stirring speed normally produces an improved drug transfer from the dosage form to the dissolution medium.

Because a best dissolution rate at the last sampling points was obtained at $100 \mathrm{rpm}$ for the basket stirrer, the performance of the basket stirrer (at $100 \mathrm{rpm}$ ) was compared to that of the paddle stirrer (at $100 \mathrm{rpm}$ ) for the FNS release. The experimental results show that the basket stirrer produced a higher dissolution percentage of the capsules than the paddle stirrer at the last sampling points (Figure 4). The basket stirrer was then selected, and it was not necessary to test the paddle stirrer at $75 \mathrm{rpm}$ and 50 rpm because these conditions would probably result in lower drug releases than at $100 \mathrm{rpm}$. The use of the basket stirrer for the dissolution test of FNS capsules complies with USP (26), which usually recommends basket stirrers as the apparatus for capsules.

The established dissolution conditions for the assessment of FNS immediate-release capsules were a dissolu-

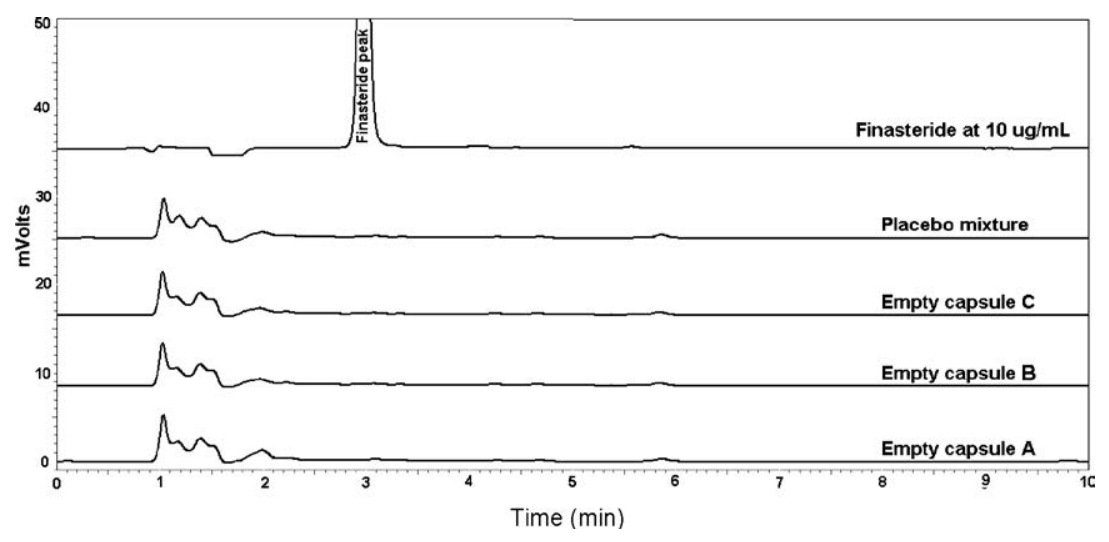

Figure 5. Chromatogram of FNS at $10.0 \mu \mathrm{g} / \mathrm{mL}$ in water, placebo mixture, and empty capsules of products $A, B$, and $C$ under dissolution conditions described in the selectivity studies.

\section{Table 3. Accuracy Studies for FNS}

\begin{tabular}{cccc}
\hline $\begin{array}{c}\text { Concentration Added } \\
(\boldsymbol{\mu} \mathbf{g} / \mathbf{m L})\end{array}$ & Day & $\begin{array}{c}\text { Concentration Found } \\
(\boldsymbol{\mu} \mathbf{g} / \mathbf{m L})\end{array}$ & $\begin{array}{c}\text { Recovery Rate } \\
(\%)\end{array}$ \\
\hline \multirow{2}{*}{5} & 1 & 4.96 & 99.21 \\
& 2 & 4.99 & 99.77 \\
& 3 & 4.94 & 98.84 \\
\hline \multirow{2}{*}{10} & 1 & 10.03 & 100.28 \\
& 2 & 9.91 & 99.12 \\
& 3 & 9.90 & 98.95 \\
\hline \multirow{2}{*}{15} & 1 & 14.91 & 99.37 \\
& 2 & 14.85 & 98.99 \\
& 3 & 14.80 & 98.65 \\
\hline
\end{tabular}

tion medium of $500 \mathrm{~mL}$ of water at $37^{\circ} \mathrm{C}$ and the basket stirrer at a stirring speed of $100 \mathrm{rpm}$. As observed in Figure 4 , these conditions produced slow drug dissolution at the first sampling points and a satisfactory dissolution at the last sampling point.

\section{Method Validation \\ Stability}

Standard solutions remained stable at room temperature for $24 \mathrm{~h}$, and sample solutions remained stable at room temperature for $2 \mathrm{~h}$ after the dissolution test (Table 2).

\section{Selectivity}

In the HPLC method, no additional chromatographic peaks from the placebo formulation and empty capsules were observed at the same retention time as FNS, demonstrating the selectivity of this method (Figure 5).

\section{Linearity}

The calibration equation obtained is $y=40790 x+3803.1$. The least-squares regression shows satisfactory correlation coefficients $(r=0.9999)$. The relative standard deviation of each point $(n=9)$ is less than $2 \%$. These results meet the acceptance criteria (26).

\section{Accuracy and Precision}

The values of the recoveries for FNS in all drug products shown in Table 3 are between $95.0 \%$ and $105.0 \%$, showing acceptable accuracy (26).

The results of the repeatability (intraassay) and intermediate precision (interassay) of the dissolution procedure are shown in Table 4. The relative standard deviations do not exceed $2.0 \%$, demonstrating suitable precision for the dissolution test (26). 
Table 4. Precision Study Results for FNS

\begin{tabular}{ccc}
\hline $\begin{array}{c}\text { Concentration Added } \\
(\boldsymbol{\mu} \mathbf{g} / \mathbf{m L})\end{array}$ & $\begin{array}{c}\text { Intraday Precision } \\
(\% \mathrm{RSD}, \boldsymbol{n}=\mathbf{3})\end{array}$ & $\begin{array}{c}\text { Intermediate Precision } \\
(\% \mathrm{RSD}, \boldsymbol{n}=\mathbf{9})\end{array}$ \\
\hline 5 & 0.91 & 1.82 \\
\hline 10 & 0.55 & 1.22 \\
\hline 15 & 1.29 & 1.95 \\
\hline
\end{tabular}

\section{Detection and Quantitation Limits}

The detection limit for the method is $0.096 \mu \mathrm{g} / \mathrm{mL}$. The quantitation limit is $0.32 \mu \mathrm{g} / \mathrm{mL}$. These results demonstrate that the analyses were performed at concentrations greater than these values, and that the method can be applied to capsules containing $1 \mathrm{mg}$ of the active ingredient.

\section{Robustness}

The results obtained in the Youden and Steiner robustness test are shown in Table 5. Effect values for all factors are less than the criterion SD $\sqrt{2}$. The method was therefore fairly robust for all factors considered in this study.

\section{Assay of Pharmaceutical Products}

The validated dissolution test was applied to the assessment of three different commercial products ( $A, B$ and C) labeled as containing $5 \mathrm{mg}$ of FNS. Commercial FNS capsules may contain 1 or $5 \mathrm{mg}$ of active drug substance. Although only the high dosage form was used in this study, the HPLC method is also able to quantify capsules containing $1 \mathrm{mg}$ of FNS after dissolution tests, as demonstrated in the validation studies. To estimate the performance of finasteride release from capsules with respect to the reference product, six reference tablets were placed into individual baskets, and the optimized and validated dissolution method was applied to the commercial product Proscar (5-mg tablets).

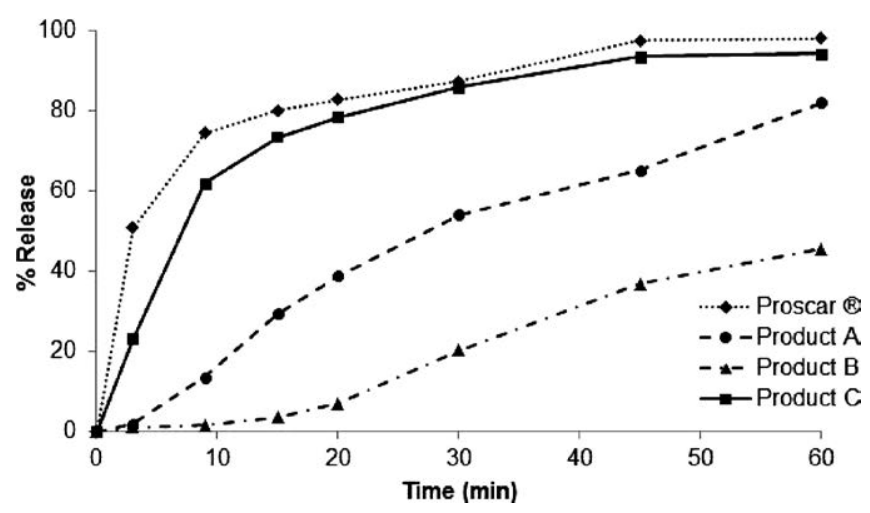

Figure 6. Dissolution profiles of 5-mg capsules of FNS products codified as $A, B$, and $C$ and the reference drug (Proscar) under optimal dissolution conditions.
Table 5. Robustness Test Results

\begin{tabular}{lcc}
\hline \multicolumn{1}{c}{ Factor } & \% Recovery & Effect (\%) \\
\hline Stirring speed & 94.2 & 2.30 \\
\hline Dissolution medium degassing & 97.1 & 1.25 \\
\hline Sample filtration & 95.1 & -2.65 \\
\hline Time of dissolution test & 93.8 & -0.90 \\
\hline Temperature of dissolution medium & 96.7 & 1.00 \\
\hline Exposure to light & 89.9 & -0.25 \\
\hline Sampling point & 94.3 & -1.00 \\
\hline SD $\sqrt{2}$ & & 3.09 \\
\hline
\end{tabular}

Dissolution profiles of the analyzed products are presented in Figure 6. The results are expressed as percentage drug release versus time ( $\mathrm{min})$. A comparison study between the commercial products and the reference product was carried out using the similarity $\left(f_{2}\right)$ factor (Table 6). The results show that capsule product $C$ had a dissolution profile closest to the reference product. Moreover, the differences observed among dissolution profiles of capsules indicate the discriminatory power of the proposed method and the necessity to establish an official dissolution method for quality control of FNS capsules.

The reference product formulation contains a surfactant, and all tests of clinical efficacy and safety were performed with this product. Thus it is proposed that all compounded capsules should contain a surfactant to improve release, primarily because FNS is a Class 2 drug in the Biopharmaceutics Classification System (low solubility/high permeability drug), where drug dissolution may be the rate-limiting step for drug absorption.

\section{CONCLUSION}

In this work, a dissolution test for FNS in capsules was optimized. The established dissolution conditions were $500 \mathrm{~mL}$ of water at $37 \pm 0.5^{\circ} \mathrm{C}$ (a "first choice" dissolution medium) and a basket at a stirring speed of $100 \mathrm{rpm}$. The validation results demonstrate that all the data met the acceptance criteria. A comparison with the three commercial products shows the ability of the method to differentiate among the tested pharmaceutical formulations. Since there is no monograph for FNS in capsules, this study illustrates the importance of an official method for the dissolution test of FNS capsules. Moreover, standardization

Table 6. Difference and Similarity Factors for the Reference Product and Products $A, B$, and $C$

\begin{tabular}{lc}
\hline Product & Similarity Factor $\left(f_{2}\right)$ \\
\hline Reference vs. A & -4.51 \\
\hline Reference vs. B & -13.67 \\
\hline Reference vs. C & 23.18 \\
\hline
\end{tabular}


of excipients is needed to ensure that marketed capsules provide the same quality as the reference product, which was subjected to rigorous tests.

\section{Acknowledgments}

The authors are grateful to the Center of Pharmaceutical Equivalence-Nucleus Quality Control for technical support.

\section{References}

1. Dissolution Testing of Immediate Release Solid Oral Dosage Forms; Guidance for Industry; U.S. Department of Health and Human Services, Food and Drug Administration, Center for Drug Evaluation and Research (CDER), U.S. Government Printing Office: Washington, DC, 1997.

2. Vaucher, L. C.; Paim, C. S.; Lange, A. D.; Schapoval, E. E. S. Development and validation of a dissolution test for telithromycin in coated tablets. Quím. Nova 2009, 32 (5), 1329-1333.

3. Schmidt, L. J.; Tindall, D. J. Steroid $5 \alpha$-reductase inhibitors targeting BPH and prostate cancer. J. Steroid Biochem. Mol. Biol. 2011, 125 (1-2), 32-38.

4. Cha, E. K.; Shariat, S. F. The Use of $5 \alpha$-Reductase Inhibitors for the Prevention and Treatment of Prostate Cancer. Eur. Urol. 2011, 59 (4), 515-517.

5. Lucas, J. K. Finasteride in the Treatment of Hirsutism. J. Women's Health 1995, 4 (6), 655-661.

6. Mella, J. M.; Perret, M. C.; Manzotti, M.; Catalano, H. N.; Guyatt, G. Efficacy and Safety of Finasteride Therapy for Androgenetic Alopecia. Arch. Dermatol. 2010, 146 (10), 1141-1150.

7. Tully, A. S.; Schwartzenberger, J.; Studdiford, J. Androgenic Alopecia. J. Mens Health 2010, 7 (3), 270-277.

8. Yamazaki, M.; Miyakura, T.; Uchiyama, M.; Hobo, A.; Irisawa, R.; Tsuboi, R. Oral finasteride improved the quality of life of androgenetic alopecia. J. Dermatol. 2011, 38 (8), 773-777.

9. Almeida, H. M.; Cabral Marques, H. Physicochemical characterization of finasteride: PEG 6000 and finasteride:Kollidon $\mathrm{K} 25$ solid dispersions, and finasteride: $\beta$-cyclodextrin inclusion complexes. J. Incl. Phenom. Macro. 2011, 70 (3-4), 397-406.

10. Ilango, K.; Valentina, P.; Lakshmi, K. S. Spectrophotometric Method for the Estimation of Finasteride in Tablets. Indian J. Pharm. Sci. 2002, 64 (2), 174-175.

11. Ilango, K.; Valentina, P.; Lakshmi, K. S. Spectrophotometric determination of Finasteride in tablet formulation. Indian Drugs 2003, 40 (2), 122-123.

12. Ulu, S. T. A new spectrophotometric method for the determination of finasteride in tablets. Spectrochim. Acta Part A 2007, 67 (3-4), 778-783.

13. Meyyanathan, S. N.; Ramasarma, G. V. S.; Suresh, B. Analysis of finasteride in pharmaceutical preparations by high performance thin layer chromatography. J. Planar Chromatogr.-Mod. TLC 2001, 14 (3), 188-190.

14. Patel, D. B.; Patel, N. J. Validated RP-HPLC and TLC methods for simultaneous estimation of tamsulosin hydrochloride and finasteride in combined dosage forms. Acta Pharm. (Zagreb, Croatia) 2010, 60 (2), 197-205.

15. Ryan, J. A.; Compton, S. V.; Brooks, M. A.; Compton, D. A. C. Rapid verification of identity and content of drug formulations using mid-infrared spectroscopy. J. Pharm. Biomed. Anal. 1991, 9 (4), 303-310.

16. Amer, S. M. Polarographic behavior and determination of finasteride. Farmaco 2003, 58 (2), 159-163.

17. Álvarez-Lueje, A.; Brain-Isasi, S.; Núñez-Vergara, L. J.; Squella, J. A. Voltammetric reduction of finasteride at mercury electrode and its determination in tablets. Talanta 2008, 75 (3), 691-696.

18. Sağlic, S.; Ulu, S. T. Development and validation of a new gas flame ionization detector method for the determination of finasteride in tablets. Anal. Biochem. 2006, 352 (2), 260-264.

19. Trapani, G.; Dazzi, L.; Pisu, M. G.; Reho, A.; Seu, E.; Biggio, G. A rapid method for obtaining finasteride, a $5 \alpha$-reductase inhibitor, from commercial tablets. Brain Res. Protoc. 2002, 9 (2), 130-134.

20. Syed, A. A.; Amshumali, M. K. LC determination of finasteride and its application tostorage stability studies. J. Pharm. Biomed. Anal. 2001, 25 (5-6), 10151019.

21. Segall, A. I.; Vitale , M. F.; Perez, V. L.; Palacios, M. L.; Pizzorno, M. T. A stability indicating HPLC method to determine finasteride in a tablet formulation. J. Liq. Chromatogr. Relat. Technol. 2002, 25 (20), 3167-3176.

22. Demir, H.; Cucu, A.; Sakarya, S. Determination of finasteride in the tablet form by liquid chromatography and its analytical method validation. Anal. Chim. Acta. 2006, $557(1-2), 252-255$.

23. Basavaiah, K.; Somashekar, B. C. Determination of Finasteride in Tablets by High Performance Liquid Chromatography. E-J. Chem. 2007, 4 (1), 109-116.

24. Fekete, S.; Fekete, J.; Ganzler, K. Shell and small particles; Evaluation of new column technology. J. Pharm. Biomed. Anal. 2009, 49 (1), 64-71.

25. Fekete, S.; Fekete, J.; Ganzler, K. Characterization of new types of stationary phases for fast liquid chromatographic applications. J. Pharm. Biomed. Anal. 2009, 50 (5), 703-709.

26. The United States Pharmacopeia and National Formulary USP 34-NF 29; The United States Pharmacopeial Convention, Inc.: Rockville, MD, 2011.

27. International Conference on Harmonisation of Technical Requirements for Registration of Pharmaceuticals for Human Use. Validation of Analytical Procedures: Text and Methodology, Q2(R1); ICH Harmonised Tripartite Guideline: Geneva, Switzerland, 2005. http://www.ich.org/fileadmin/Public_Web_Site/ 
ICH_Products/Guidelines/Quality/Q2_R1/Step4/Q2 R1_Guideline.pdf (accessed June 19, 2013).

28. Donato, E. M.; Martins, L. A.; Fröehlich, P. E.; Bergold, A. M. Development and validation of dissolution test for lopinavir, a poorly water-soluble drug, in soft gel capsules, based on in vivo data. J. Pharm. Biomed. Anal. 2008, 47 (3), 547-552.
29. Youden, W. J.; Steiner, E. H. Statistical Manual of the AOAC. AOAC International: Gaithersburg, MD, 1975.

30. Menegola, J.; Steppe, M.; Schapoval, E. E. S. Dissolution test for citalopram in tablets and comparison of in vitro dissolution profiles. Eur. J. Pharm. Biopharm. 2007, 67 (2), 524-530. 